\title{
Post-chemotherapy bone metastasis recurrence of colorectal adenocarcinoma - case report
}

\author{
Răzvan Ene***, Marian Pleniceanu*, Mihnea Popa***, Mihai Nica***, Panti Zsombor***, \\ Bogdan Şerban*, Cătălin Cîrstoiu* ** \\ *Orthopedics and Traumatology Department, Emergency University Hospital, Bucharest, Romania \\ **"Carol Davila" University of Medicine and Pharmacy, Bucharest, Romania
}

Correspondence to: Marian Pleniceanu, MD, PhD,

Orthopedics and Traumatology Department, Emergency University Hospital, Bucharest, Romania, 169 Independentei Street, Code 050098, Bucharest, Romania, Mobile Phone: +40769603 041, E-mail: marian3006@yahoo.com

\begin{abstract}
One of the most common neoplasms in the world, ranking third, is the colorectal cancer. $96 \%$ of all cases of colorectal cancer are adenocarcinoma. The prognostic of this diagnostic is a bad one, with low 5 -years survival rate. Although the most common locations for the metastases are the liver and the lungs, the bones can also be targeted (5.5\%).

We present the case of a 65 -year-old male who came to the hospital with pain and tumefaction at the right fibular head. After extensive clinical and paraclinical examinations, a biopsy was performed, at which stage the entire tumor was removed. The result was that of moderate differentiated adenocarcinoma. The patient was then further examined and a tumor was discovered at the sigmoid colon. The primary tumor was removed and the patient underwent oncological treatment. 2 years later, the patient returned with a recurrence of the bone metastasis. After a PET-CT that infirmed other metastases coupled with a satisfactory evolution of the primary tumor, the decision was made to amputate the knee above. Biopsy is an essential step that establishes the correct diagnostic and dictates the right choice of treatment solution. Counseling is also essential, so that the patient can choose and accept the best treatment option. Keywords: biopsy, amputation, prognosis
\end{abstract}

\section{Introduction}

The colorectal cancer is one of the most common types of cancer worldwide, ranking third, with an estimated 1.2 million new cases diagnosed worldwide every year. $96 \%$ of all colorectal cancers are adenocarcinoma. Studies in the US show that approximately $4.6 \%$ of men and $4.2 \%$ of women will be diagnosed with CRC in their lifetime $[14,15]$.
The distribution between men and women is close to equal with a slight incline to the male gender. The median age of diagnosis for colon cancer is 68 years for men and 72 years for women, and a median age of 63 years for rectal cancer for both men and women $[11,19,20]$. The risk factors for developing colorectal cancer are multiple and various, ranging from genetic (specific gene mutations that associate other syndromes, such as Lynch syndrome, 
familial adenomatous polyposis, with a higher risk for colorectal cancer) to personal medical history (chronic inflammatory bowel disease, diabetes) to behavioral factors (physical inactivity, overweight and obesity, diet, smoking, alcohol) $[7,12,21]$. The treatment options depend on the stage of the cancer and can be curative or palliative. When discovered in late stages, it is imperative to search for secondary determinations. The most common sites for metastases are the liver and the lungs, but other locations can be affected, such as the peritoneum, the ovaries, and in some cases, the bones [1-3].

\section{Case report}

In January 2015, a 65-year-old patient came to our Clinic at the Emergency University Hospital of Bucharest with pain and a tumefaction matching the site of the right fibular head. The patient stated that the tumefaction grew over a period of 6 months. However, the pain only began approximately 1 month before the patient went to the hospital. The lack of any recent traumatic history and the growing tumefaction coupled with the pain that did not subside with any anti-inflammatory treatment, urging the patient to go to the hospital. Upon arrival, a preliminary set of investigations were made (blood tests and a simple right knee $X$-ray). The X-ray showed a tumoral growth of the fibular head. This was followed by the minute investigation of the patient (Fig. 1 CT, Fig. 2 MRI, bone scintigraphy).

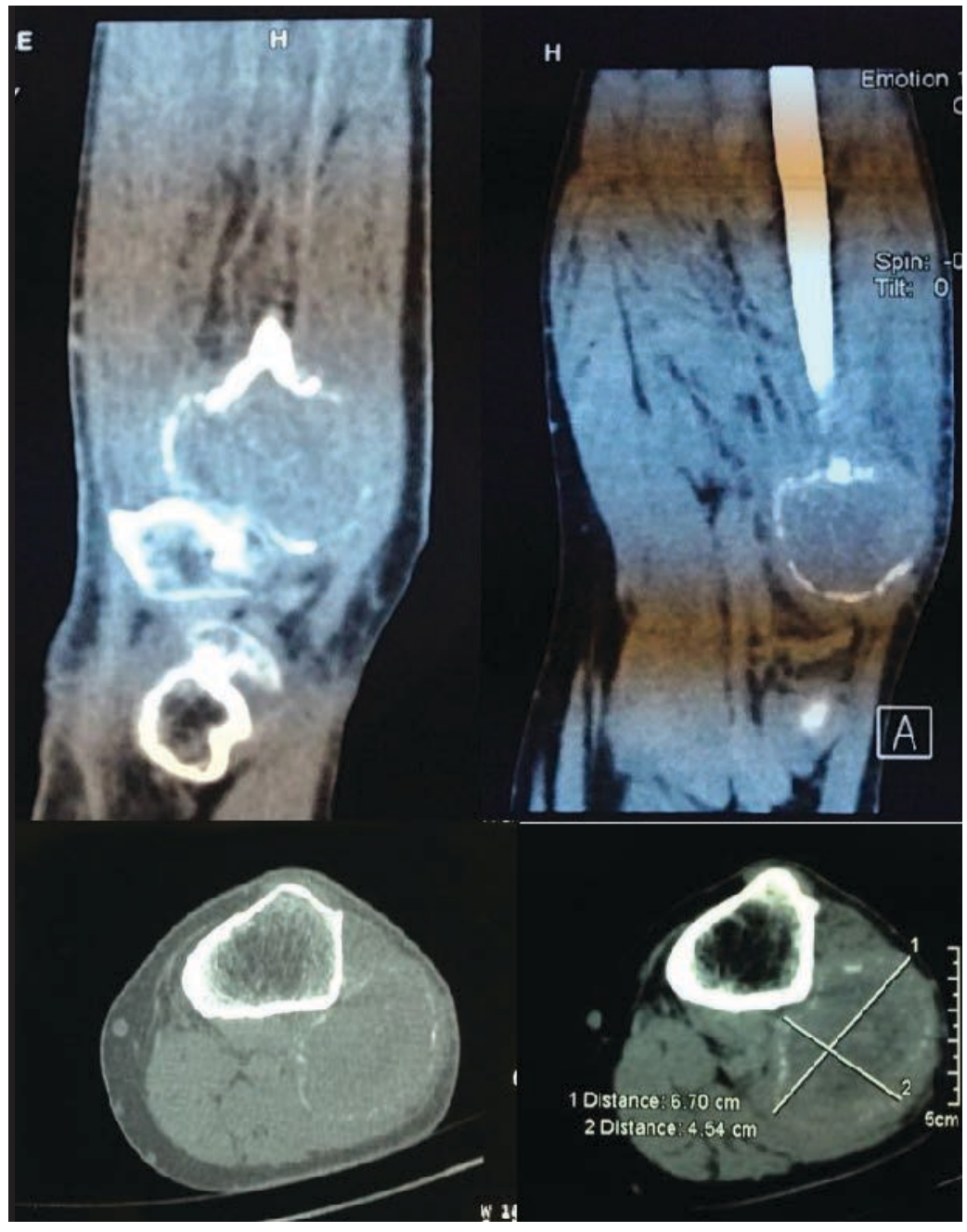

Fig. 1 CT sections of the fibular head metastasis

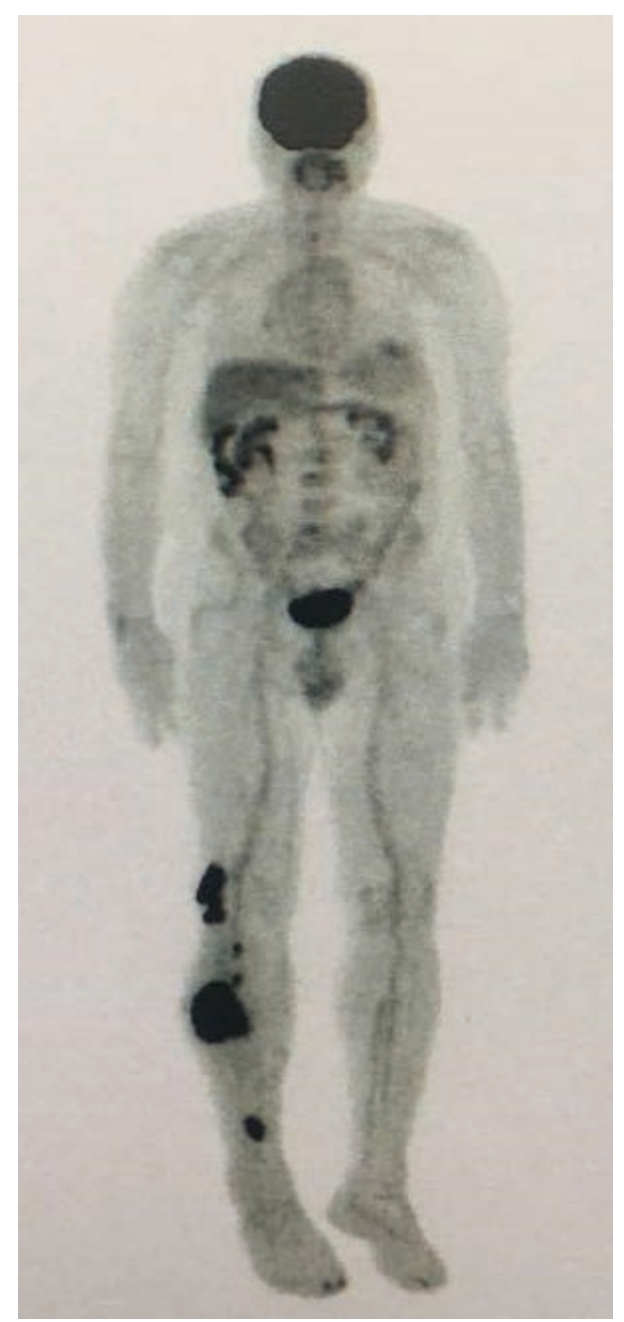

Fig. 2 Bone scintigraphy 
After careful planning and preparation, a biopsy was performed in January 2015 (Fig. 4). During the surgery, the entire tumor was removed. The histopathology result that arrived was strange, as it was indicating a moderate differentiated adenocarcinoma. Upon learning this result, the patient was scheduled for a whole body CT-scan that revealed a tumor of the sigmoid colon (Fig. 3).

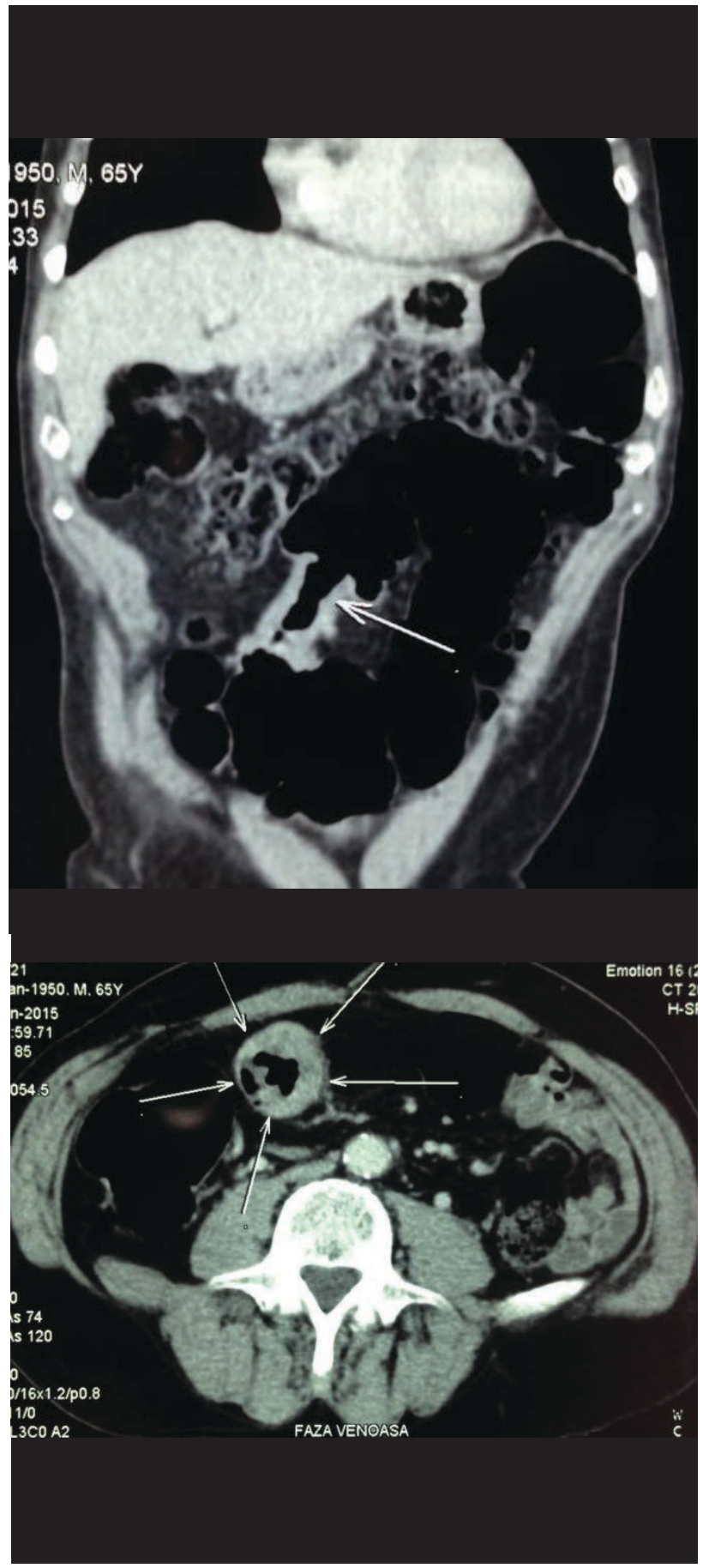

Fig. 3 Abdominal CT-scan showing the sigmoid colon tumor

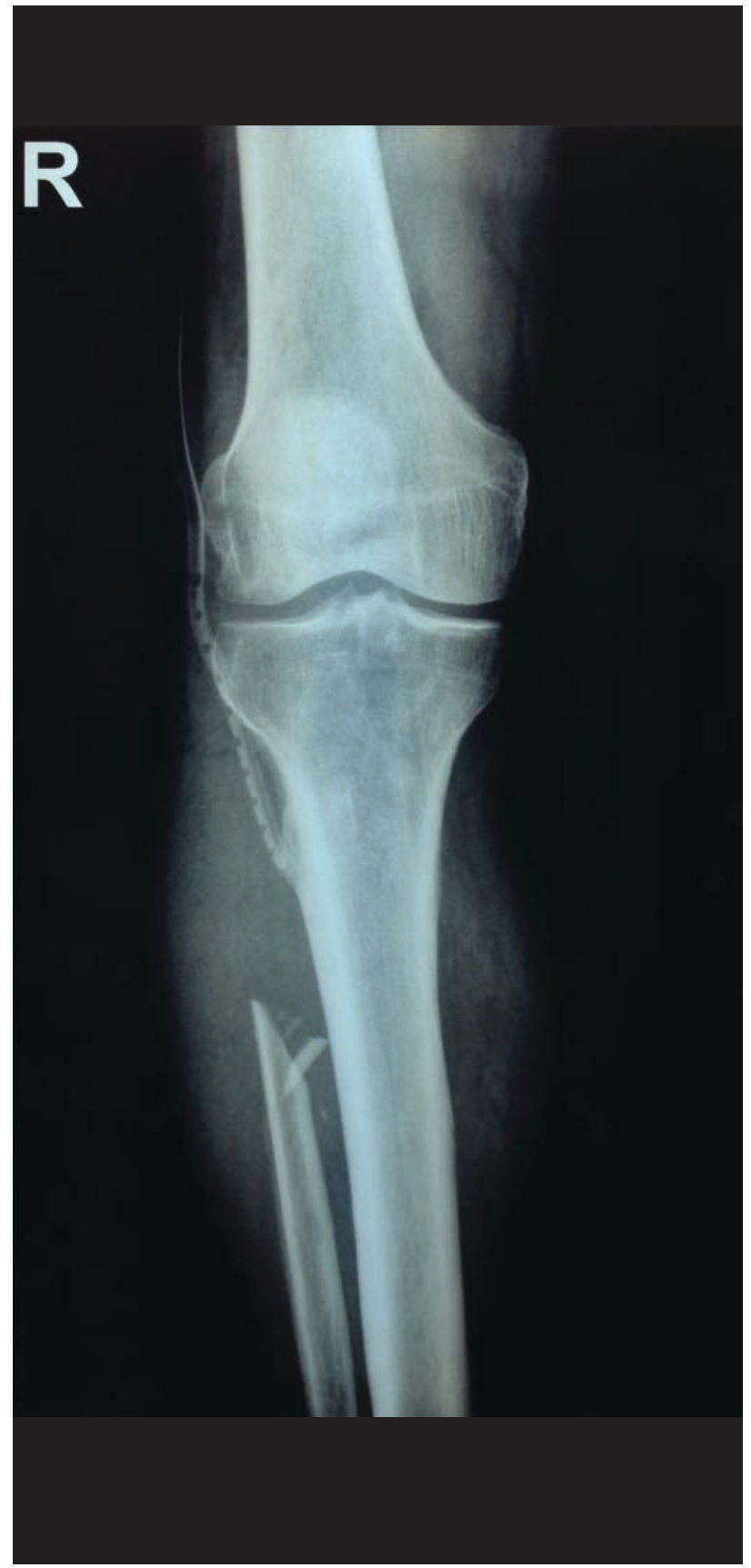

Fig. 4 Post-surgery X-ray after biopsy

The patient was subjected to surgery and the colon tumor was removed. Subsequently, he started chemotherapy in the Oncological Department of our Hospital. There, he was subjected to chemotherapy with a personal treatment with 5-fluorouracil, leucovorin, and oxaliplatin. After a 2-year period, the patient returned to our Clinic with a tumefaction on the biopsy site. An X-ray of the right knee showed the recurrence of the tumor, with a bigger volume than the previous lesion (Fig. 5). 


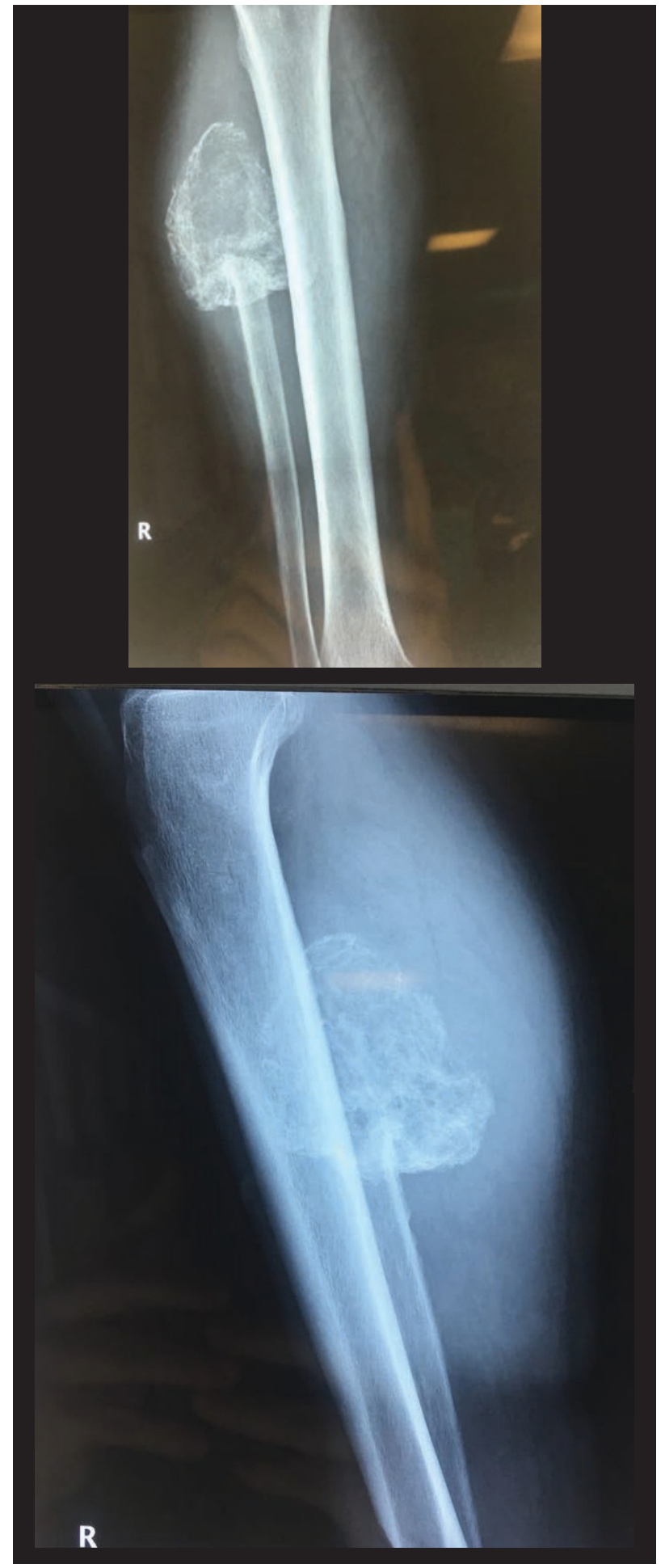

Fig. 5 X-rays highlighting the recurrence of the metastasis

At that respective moment, the patient was still undergoing the oncological treatment. A PET-CT was performed and the result infirmed other metastases. After interdisciplinary consulting, the decision was made to amputate from the distal femur. The reasoning for this decision was the absence of other metastases, the favorable evolution of the primary tumor site, as well as the highest survival rate chance. The patient was informed of all aspects and gave his consent for the surgery. In November 2017, the amputation of the distal femur was performed. Post-surgery, the evolution was favorable. The follow-ups were at 12 days, 6 weeks, 3 months and are still ongoing.

\section{Discussions}

The particularity of this case was the recurrence of the metastasis although chemotherapy was administered. The bone metastasis of a colorectal cancer is in itself a rare location, but this was not always the case. Before the introduction of modern chemotherapy, bone metastases were reported in $10-24 \%$ of colorectal cancers $[22,23]$. Nowadays, this percentage is lower (5.5\%), but still controversial. The overall survival period was raised with said new chemotherapy drugs and targeted treatments, and thus the chance for a bone metastasis to develop also increased. The location of the metastasis was also a particularity of this case, the most common site for this kind of neoplasm being the spine $(65 \%)$, followed by the pelvis, skull, and then the long bones $[4,6,8,9]$. The prognosis for bone metastases in colorectal cancer is bad, with studies showing that the 5 -year survival rate is $8.1 \%$, with a median survival rate of 16 months [16-18].

Another important aspect is the psychological one. When the average person is faced with the diagnosis of a cancer, the psychological implications are vast. Studies have shown that $87 \%$ of the patients develop a form of depression. The discovery of a secondary site further adds stress and tension. Many of them choose to give up and refuse more radical treatment that may prolong or even save their lives. Counseling is imperative, so that the patient can cope better with the reality of this disease, and all that it implies, including the hard decisions that have to be made $[5,10,13]$. 


\section{Conclusions}

The most important thing, when up against a tumoral growth, is for the surgeon to do a biopsy of the said tumor. This essential step will determine the correct diagnosis as well as the right choice of treatment.

Another crucial step before choosing any type of treatment is to first treat the primary tumor that gave birth to the metastasis. The evolution of the primary tumor establishes the type of treatment for the subsequent metastases, be it curative if the evolution is good or palliative if it is not.

Last, but not least, all physicians should offer moral support, because patients faced with these diagnoses tend to make wrong decisions that have a potentially vital impact.

\section{References}

1. Cirstoiu C, Ene R. Examenul clinic in ortopedie. 2013, Bucuresti, Editura Universitara Carol Davila.

2. Ene R, Sinescu R, Ene P, Popescu D, Cirstoiu M, Cirstoiu C. Proximal tibial osteosarcoma in young patients: early diagnosis. Modular reconstruction. Romanian Journal of Morphology and Embryology. 2014; 4.

3. Rodrigues J, Ramani A, Mitta $\mathrm{N}$ et al. A rare case of colon cancer with metastases to the bone with review of the literature. The Internet Journal of Oncology. 2012; 8.

4. Chambers A, Groom A, MacDonald I. Dissemination and growth of cancer cells in metastatic sites. Nature. $2 \mathrm{Au}-$ gust 2002; 563-672.

5. Liang H, Wang XN, Wang BG et al. Prognostic factors of young patients with colon cancer after surgery. World J Gastroenterol. 2006; 12:1458-62.

6. Ihle PM, McBeath AA. Bone metastasis from colonic carcinoma. A case report. J Bone Joint Surg Am. 1973; 55:398-400.

7. Daldrup-Link HE, Franzius C, Link TM et al. Wholebody MR imaging for detection of bone metastases in children and young adults: comparison with skeletal scintigraphy and FDG PET. AJR Am J Roentgenol. 2001; 177:229-36.

8. Delva R, Pein F, Lortholary A et al. Bone metastases of colorectal cancers: apropos of 8 cases. Rev Med Interne. 1993; 14:223-8.

9. Nozue M, Oshiro Y, Kurata M et al. Treatment and prognosis in colorectal cancer patients with bone metastasis. Oncol Rep. 2002; 9:109-12.

10. Nakamoto Y, Cohade C, Tatsumi M, et al. CT Appearance of Bone Metastases Detected with FDG PET as Part of the Same PET/CT Examination. Radiology 2005;237:627-
34.

11. Galasko CS. Diagnosis of skeletal metastases and assessment of response to treatment. Clin Orthop. 1995; 312:64-75.

12. Mundy GR. Metastasis to bone: causes, consequences and therapeutic opportunities. Nat Rev Cancer. 2002; 2:584-93.

13. Patanaphan V, Salazar OM. Colorectal cancer: metastatic patterns and prognosis. South Med J. 1993; 86:38-41.

14. Coleman RE. Skeletal complications of malignancy. Cancer. 1997; 80:1588-94.

15. Santini D, Tampellini M, Vincenzi B et al. Natural history of bone metastasis in colorectal cancer: final results of a large Italian bone metastases study. Ann Oncol. 2012; 23:2072-7.

16. Hoehn JL, Ousley JL, Avecilla CS. Occult carcinoma of the colon and rectum manifesting as osseous metastasis. Dis Colon Rectum. 1979; 22:129-32.

17. Weitz J, Kienle P, Magener A et al. Detection of disseminated colorectal cancer cells in lymph nodes, blood and bone marrow. Clin Cancer Res. 1999; 5:1830-36.

18. Kerr DJ, Domingo E, Kerr R. Is sidedness prognostically important across all stages of colorectal cancer?. Lancet Oncol. 2016; 17:1480-1482.

19. Yin D, Morris CR, Bates JH, German RR. Effect of misclassified underlying cause of death on survival estimates of colon and rectal cancer. J Natl Cancer Inst. 2011; 103:1130-1133.

20. Peters U, Hutter CM, Hsu L et al. Meta-analysis of new genomewide association studies of colorectal cancer risk. Hum Genet. 2012; 131:217-234.

21. Liang PS, Chen TY, Giovannucci E. Cigarette smoking and colorectal cancer incidence and mortality: systematic review and meta-analysis. Int J Cancer. 2009; 124:24062415.

22. Roth ES, Fetzer DT, Barron BJ et al. Does colon cancer ever metastasize to bone first? a temporal analysis of colorectal cancer progression. BMC Cancer. 2009; 9:274.

23. Sundermeyer ML, Meropol NJ, Rogatko A et al. Changing patterns of bone and brain metastases in patients with colorectal cancer. Clin Colorectal Cancer. 2005; 5:108-113. 